\title{
Analysis of warm-up effects during avoidance in the Mongolian gerbil
}

\author{
ROBERT W. POWELL and LINDA PALM \\ University of South Florida, Tampa, Florida 33620
}

\begin{abstract}
Mongolian gerbils were trained under unsignaled free-operant avoidance until performance stabilized. They showed slight warm-up effects, receiving approximately $40 \%$ of the total session shocks in the initial one-third of the session. Leverpressing was then studied during 1-h extinction periods as a function of the duration of an avoidance period which immediately preceded it. Responding during extinction showed only a small increase as the duration of the avoidance period increased from 1 to $60 \mathrm{~min}$. The majority of gerbils showed nonsignificant correlations between the number of extinction responses and avoidance time, avoidance responses, and shocks. The results appear to support an explanation of warm-up which emphasizes differences in activation/arousal between domesticated and nondomesticated animals.
\end{abstract}

The "warm-up effect" refers to a progressive improvement in the subject's performance over the course of the experimental session. Warm-up during freeoperant avoidance conditioning is seen as a persistent tendency by the subject to take a disproportionate number of shocks early in the session. Most domesticated rats show this pattern indefinitely, regardless of their terminal performance during the preceding session.

A number of studies in this laboratory have shown that nondomesticated rodents typically display much less warm-up during unsignaled free-operant avoidance than do domesticated rats (Powell, 1971, 1972, 1976; Powell \& Mantor, 1970; Powell \& Peck, 1969). The species studied have included Mongolian gerbils (Meriones unguiculatus), cotton rats (Sigmodon hispidus), wood rats (Neotoma floridana), and black rats (Rattus rattus). As an example of the difference between domesticated and nondomesticated rodents, we observed that hooded rats and albino rats received approximately $55 \%, 25 \%$, and $20 \%$ of total session shocks during successive thirds of the session, whereas the results for Mongolian gerbils and black rats were roughly $40 \%$, $29 \%$, and $31 \%$, respectively (Powell, 1972). Absolute shock rates were not substantially different for the various species studied.

Most attempts to explain warm-up have stressed either emotionality (Hoffman, Fleshler, \& Chorny, 1961) or activation/arousal (Powell, 1972). The former authors argue that exposure to a certain amount of shock is necessary in order to establish a supporting motivational state for avoidance behavior, and that warm-up is simply a reflection of this process. This interpretation of warm-up is consonant with two-factor theories of avoidance which stress conditioned fear as

Reprints may be obtained from Robert W. Powell, Department of Psychology, University of South Florida, Tampa, Florida 33620. a factor mediating performance (Mowrer \& Lamoreaux, 1946). Our explanation of warm-up in terms of activation/arousal refers to alertness and responsiveness to salient environmental stimuli. It seems plausible that wild species may maintain higher levels of activation/ arousal than domesticated animals because this characteristic is selected for in the natural environment, whereas no similar selective pressure exists for domesticated animals. An animal in the wild that was not particularly alert would appear to be at a competitive disadvantage. This would seem to be most true for species subject to heavy predation, which is the case with most small rodents. While gerbils have been bred in captivity for approximately 20 years (Monroe, 1967), the domestication of rats has been underway for a considerably longer period (Barnett, 1963). Thus, if activation/arousal is selected for in the wild, it should still be more prominent in gerbils than in domesticated rats.

In an earlier study which sought to examine the constituents of warm-up, we found that responding of albino rats during extinction was systematically influenced by the duration of an immediately preceding avoidance period (Powell, 1972). Significant correlations were obtained for most animals between extinction responses and avoidance time, avoidance responses, and shocks. These results appeared to support a motivational explanation of warm-up.

The present experiment was undertaken to systematically replicate the experiment described above, using the gerbil in place of the domesticated rat. Because of the slight warm-up displayed by gerbils during avoidance, we anticipated that nonsignificant correlations between the independent and dependent variables would be found. That is, because gerbils appear to maintain high levels of activation, the occurrence of shocks does not serve to boost activation to a more optimal level. Consequently, gerbils should respond approxi- 
mately the same number of times during extinction, regardless of the duration of the preceding period of avoidance. The experiment specifically tested that prediction.

\section{METHOD}

\section{Subjects}

Eight male Mongolian gerbils (Meriones unguiculatus) approximately 6 months of age were used. All subjects had been born and raised in the laboratory and were experimentally naive. The gerbils were housed in individual home cages where they had free access to food and water.

\section{Apparatus}

The gerbils were studied in a Lehigh Valley small-animal test chamber (Model 1316), $19.7 \times 20.3 \times 22.9 \mathrm{~cm}$, with a small lever at the right side of one end, $3.5 \mathrm{~cm}$ above the grid floor. Electric shock was provided by a Lehigh Valley constant-current shock source (Model 1531) and was scrambled to the grid floor of the test chamber. Data were recorded by digital counters and a Gerbrands cumulative recorder.

\section{Procedure}

Avoidance training. A free-operant avoidance procedure (Sidman, 1953) was used. Under this procedure, brief electric shocks were delivered every $5 \mathrm{sec}$ (shock-shock interval) in the absence of a response. Each leverpress postponed the next shock for $20 \mathrm{sec}$ (response-shock interval). Shock duration was $.5 \mathrm{sec}$. Responses in the presence of shock did not terminate it. Shock intensity was $1.5 \mathrm{~mA}$. Experimental sessions were $90 \mathrm{~min} /$ day. Avoidance training continued for each subject until performance stabilized. The stability criterion was a variation in the number of shocks received within a range of 20 over five consecutive sessions, that is, the largest number of shocks in any of the five sessions could not exceed the fewest shocks by more than 20 .

Extinction. Following stable avoidance, each gerbil was studied in combined avoidance-extinction sessions. Each session began with the avoidance procedure in effect for $1,5,15$, or $60 \mathrm{~min}$ followed by $60 \mathrm{~min}$ with no shocks presented. No signal was presented to indicate the change in procedure. The sequence of avoidance periods varied unsystematically within each block of five sessions. The purpose of this procedure was to eliminate temporal cues which might enable the animal to discriminate the change to extinction. All gerbils were studied for a minimum of five five-session blocks. Four gerbils showed relatively stable extinction responding across these blocks, while the other animals showed a marked decrease in extinction responding from Block 1 to 2. Data from the first block were considered to be transitional and were eliminated from the analysis. An additional block of five sessions was conducted for the latter animals.

Table 1

Total Number of Sessions to Criterion and Mean Rate of Responses and Shocks During Final Five Avoidance Sessions for Each Subject

\begin{tabular}{cccc}
\hline Subject & $\begin{array}{c}\text { Number of } \\
\text { Sessions }\end{array}$ & $\begin{array}{c}\text { Responses } \\
\text { Per Hour }\end{array}$ & $\begin{array}{c}\text { Shocks } \\
\text { Per Hour }\end{array}$ \\
\hline G-3 & 31 & 707 & 39 \\
G-5 & 28 & 487 & 34 \\
G-6 & 23 & 543 & 23 \\
G-0 & 16 & 581 & 37 \\
G-26 & 21 & 561 & 31 \\
G-27 & 15 & 1117 & 15 \\
G-29 & 9 & 546 & 15 \\
G-30 & 16 & 535 & 39 \\
Mean & 20 & 635 & 29 \\
\hline
\end{tabular}

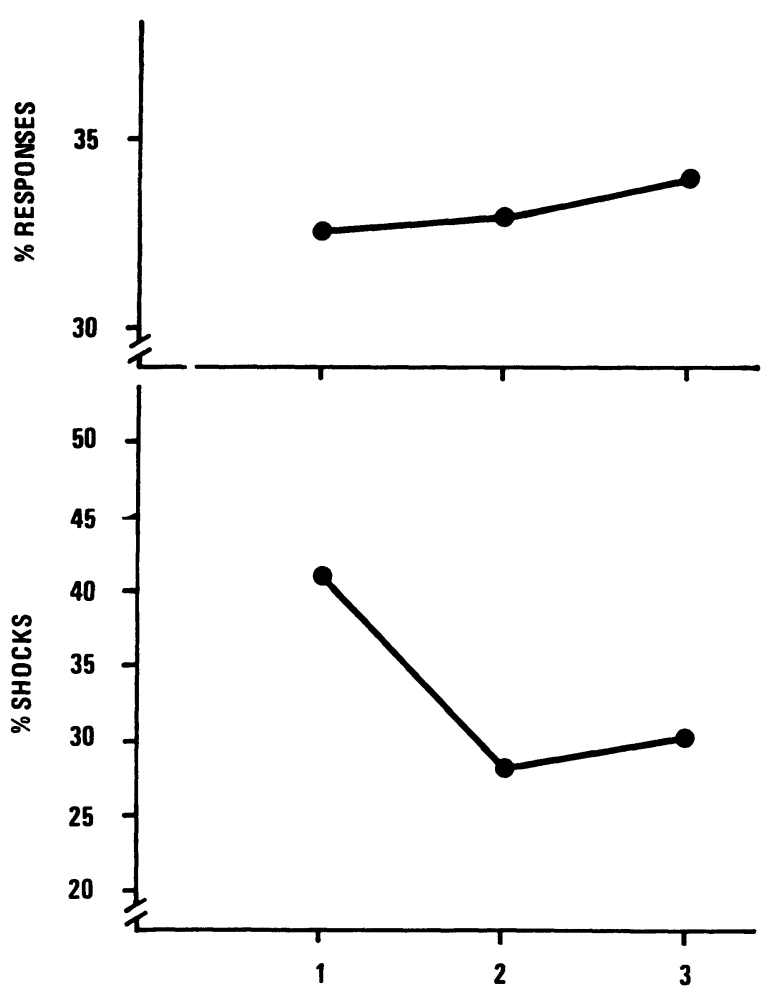

THIRDS OF AVOIDANCE SESSION

Figure 1. The percentage of responses and shocks during successive 30-min intervals of the avoidance session.

\section{RESULTS}

\section{Avoidance}

All of the gerbils learned to avoid effectively. Hourly response rates and shock rates, which are shown in Table 1, are quite similar to those reported earlier for gerbils studied under similar conditions (Powell, 1971, 1972). Warm-up effects were analyzed by dividing experimental sessions into thirds ( $30 \mathrm{~min})$ and determining the number of responses and shocks in each interval. Group curves showing these distributions are presented in Figure 1. The gerbils showed a small increase in responding over the session, and received a slightly higher percentage of shocks in the initial third of the session. Thus our expectation that the gerbils would display only a minor degree of warm-up was confirmed. While the amount of warm-up displayed by the gerbils was small in comparison to domesticated rats, a repeated measures analysis of variance with subsequent tests did indicate that a significantly greater percentage of shocks was received during the initial one-third of the session $(F=6.14, d f=2 / 14, p<.01)$. There was no difference between subsequent thirds of the session. It seems noteworthy that the response and shock curves presented here are almost exact duplicates of the same curves for gerbils observed earlier (Powell, 1972). 
Table 2

Mean Avoidance Responses, Shock Rates, and Responses During Extinction as a Function of the Duration of the Preceding Avoidance Period for the Group

\begin{tabular}{lrrrrr}
\hline & \multicolumn{5}{c}{ Avoidance Time in Minutes } \\
& \multicolumn{1}{c}{1} & \multicolumn{1}{c}{2} & \multicolumn{1}{c}{15} & \multicolumn{1}{c}{30} & \multicolumn{1}{c}{60} \\
\hline Avoidance Responses/Min & 11.3 & 10.4 & 10.7 & 11.9 & 12.0 \\
Shocks/Min & 1.9 & .9 & .9 & .7 & .6 \\
Extinction Responses & 73.7 & 70.8 & 83.4 & 104.0 & 109.2 \\
\hline
\end{tabular}

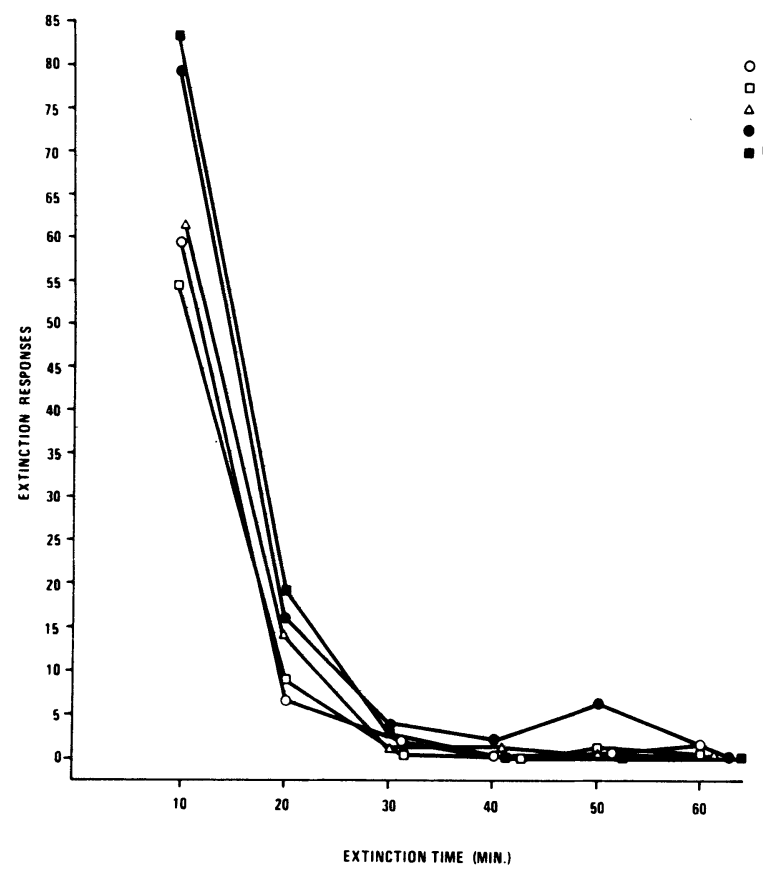

Figure 2. Extinction curves for the group as a function of the amount of time under the avoidance schedule.

\section{Extinction}

The total number of responses emitted during extinction increased slightly as the duration of the preceding avoidance period increased. Table 2 shows also that avoidance response rate was unchanged, while shock rate decreased slightly across avoidance periods. This result shows that slight warm-up continued during this part of the experiment. The relative constancy of shock rate after 5 min suggests that warm-up was complete within this period.

Figure 2 presents group curves which show the relationship between extinction time and the number of extinction responses. Responding during extinction declined rapidly after the first $10 \mathrm{~min}$ and differences in the group curves grew progressively smaller over the remainder of the extinction session. A series of Friedman analyses of variance showed that the only significant difference in extinction responding as a function of avoidance time occurred during the first $10 \mathrm{~min}$ of extinction $(\mathrm{H}=12.92, \mathrm{df}=4, \mathrm{p}<.01)$.

Table 3 presents the correlations between the three versions of the independent variable and the dependent variable. Only two of the eight gerbils showed significant correlations between two or more of the independent variables and the number of responses during extinction.

\section{DISCUSSION}

The present results support our prediction that gerbils would show little relationship between the duration of an avoidance period and the number of responses emitted during a subsequent extinction period. The gerbils' behavior also conformed to our expectation in that they showed little warm-up during the avoidance period itself, receiving approximately $40 \%$ of the total session shocks in the initial one-third of the session. Albino rats received $55 \%-60 \%$ of the total session shocks during the first one-third of the session, and they showed a much stronger relationship between the length of the avoidance period and the number of extinction responses (Powell, 1972).

A systematic comparison of the results obtained here with those of the albino rats studied earlier under similar conditions shows substantial interspecies difference. For example, extinction responses for the rats were $125 \%$ higher following a 60 -min avoidance period, in comparison to a $1-\min$ avoidance period, while the gerbils showed an increase of only $47 \%$ under the same conditions. Similarly, responding by the rats during the first $10 \mathrm{~min}$ of extinction was $161 \%$ higher following $60 \mathrm{~min}$ of avoidance vs $1 \mathrm{~min}$, while the gerbils showed an increase of only $41 \%$. Each of the five albino rats studied earlier showed significant correlations between two or more of the independent variables (avoidance time, avoidance responses, shocks) and the number of extinction responses, while only two of the eight gerbils studied here showed significant correlations.

The observed differences between domesticated and nondomesticated rodents in warm-up and responding during avoidance extinction suggest that differences in activation/arousal may be the critical factor operating. It has been argued that exposure to a certain amount of shock is necessary in (domesticated) rats in order to establish a supporting motivational state for avoidance behavior (Hoffman, Fleshler, \& Chorny, 1961). This explanation emphasizes emotionality as the principal factor mediating warm-up in avoidance. We prefer to emphasize activation/arousal as the mechanism which accounts for the differences between species. This explanation focuses upon alertness and responsiveness to salient environmental stimuli. It is preferred because of the following considerations: (1) Gross observations do not reveal any obvious differences in emotionality between domesticated and nondomesticated rodents undergoing avoidance training, as best this can be inferred. (2) It has recently been reported that wild rats show a substantial improvement under signaled free-operant avoidance in comparison to an unsignaled procedure, whereas domesticated

Table 3

Pearson Product-Moment Coefficients of Correlation Between Number of Responses During Extinction and Avoidance Time, Avoidance Responses, and Shocks

\begin{tabular}{|c|c|c|c|}
\hline \multirow[b]{2}{*}{ Subject } & \multicolumn{3}{|c|}{ Pearson Correlations } \\
\hline & $\begin{array}{c}\text { Avoidance } \\
\text { Time }\end{array}$ & $\begin{array}{l}\text { Avoidance } \\
\text { Responses }\end{array}$ & Shocks \\
\hline G-0 & .057 & .123 & -.114 \\
\hline G-3 & $.473 *$ & $.491 *$ & .268 \\
\hline G-5 & $.630 \dagger$ & $.662 \dagger$ & $.543 * *$ \\
\hline G-6 & .330 & .311 & .352 \\
\hline G-26 & .213 & .226 & -.123 \\
\hline G-27 & .154 & .236 & .077 \\
\hline G-29 & .300 & .328 & .207 \\
\hline G-30 & .029 & .059 & .021 \\
\hline .05 & ** & & $t_{p}<.001$ \\
\hline
\end{tabular}


rats show little or no improvement when the signal is added (Powell, 1976). (3) It seems plausible that animals which maintain higher levels of activation/arousal would be selected for in the natural environment, whereas no similar selective pressure exists in the laboratory.

\section{REFERENCES}

Barnett, S. A. The rat. Chicago: Aldine, 1963.

Hoffman, H. S., Fleshler, M., \& Chorny, H. Discriminated barpress avoidance. Journal of the Experimental Analysis of Behavior, 1961, 4, 309-316.

Monroe, B. N. Gerbils. Jersey City, N.J: TFH, 1967.

Mowrer, O. H., \& LamoreaUX, R. R. Fear as an intervening variable in avoidance conditioning. Journal of Comparative and Physiological Psychology, 1946, 39, 29-50.

Powell, R. W. Free-operant (Sidman) avoidance in fieldraised and laboratory-raised wild rats. Journal of
Comparative and Physiological Psychology, 1971, 75, 216-225.

Powell, R. W. Analysis of warm-up effects during avoidance in wild and domesticated rodents. Journal of Comparative and Physiological Psychology, 1972, 78, 311-316.

Powell, R. W. A comparison of signaled vs unsignaled freeoperant avoidance in wild and domesticated rats. Animal Learning \& Behavior, 1976, 4, 279-286.

Powell, R. W., \& MantoR, H. Shaping of free-operant avoidance in the wood rat Neotoma floridana. Psychonomic Science, 1970, 20, 263-265.

Powell, R. W., \& PECK, S. Activity and avoidance in the Mongolian gerbil. Journal of the Experimental Analysis of Behavior, 1969, 12, 779-787.

Sidman, M. Two temporal parameters of the maintenance of avoidance behavior by the white rat. Journal of Comparative and Physiological Psychology, 1953, 46, 253-261.

(Received for publication December 4, 1976.) 